\title{
Ureteroscopy Holmium Laser Lithotripsy Treatment Complicated Ureteral Stones
}

\author{
Fan-ping MENG ${ }^{1, a}$, Jun WEI ${ }^{1}$, Yong-liang $\mathrm{CUI}^{1}$ and Ji GAO ${ }^{1, \mathrm{~b},{ }^{*}}$ \\ ${ }^{1}$ Department of Urology, China-Japan Union Hospital, Jilin University, \\ Changchun, Jilin, China \\ a947410581@qq.com, b1217760@qq.com \\ ${ }^{*}$ Corresponding author
}

Keywords: Ureteral soft mirror combined ureteral hard mirror, Holmium laser lithotripsy, Complex ureteral stones in the ureter.

\begin{abstract}
Objective: to discuss the effectiveness and safety of the ureteral stone in complicated ureteroscope of ureteroscope. Methods: in this study, 47 cases of ureteral calculi were treated by the ureteroscope of the ureteroscope, which was collected from February 2015 to February 2017. Results: in 47 cases, 43 cases of single operation were successful, and the rate of single operation stone crushing was 91.5\% (43/47). 1 case of ureteral perforation when entering the mirror; One case of the ureteral was heavily distorted to open surgery; Two cases of soft mirror did not reach the stone directly, leave the F6 double J tube, and two weeks later, the ureter was broken. Conclusion: the ureteral soft holmium laser lithotripsy combined with ureter mirror mirror on the complexity of ureteral Duan Jieshi safe and effective treatment, curative effect is a kind of exact intracavitary urology macadam method.
\end{abstract}

\section{Introduction}

Complexity of ureteral Duan Jieshi because in ureteral stay inside for a long time, its surrounding tissues can happen relatively significant inflammation, and formation of polyps or calculus incarcerated, parcels, can result in ureteral obstruction, if you don't remove the obstruction in time, will lead to different degree of kidney damage[1]. Our hospital from February 2015 to February 2015 using fibre soft ureter mirror with rigid ureteroscopy holmium laser therapy on the complexity of ureteral Duan Jieshi 47 cases, curative effect is satisfied, now report as follows.

\section{Data and Methods}

General data: 27 men and 20 women in the group; Age 19-68, average 41.6; The main symptom of clinical symptoms is the side pain; The duration of the disease was 5 days 24 months, on average (4.46 plus or minus 1.42) months. Location of stone: 24 cases on the right, 23 on the left, 3 in bilateral ureteral stones. All the ureteral stones were in the ureteral tube, in which the ureteral stricture was narrowed or distorted 16 cases, with a narrow length of 0.5 to $2.0 \mathrm{~cm}$. Combine polyps or granulates in 40 cases. The size of a stone is $0.5 \mathrm{~cm}$ by $0.6 \mathrm{~cm}-2.0 \mathrm{~cm} \times 2.2 \mathrm{~cm}$. The preoperative $\mathrm{WBC}$ value is controlled at $0-15$ / HP. The urinary tract $\mathrm{B}$ overshowed 17 cases of the lateral renal pelvis and 30 cases of moderate water. Nine patients were treated 1-3 times before the operation, including 4 in stone and 5 in situ.

Diagnostic criteria: a stone stays in the ureter for $>8$ weeks and the maximum size of $>$ $0.8 \mathrm{~cm}$. The IVU contrast agent cannot be separated from the stone, or from the side of 
the pelvis. The following ureteral tubes are distorted or narrow; The site of the stone or the following ureteral polyp form or stone inlaid with the ureter is tightly bonded to the ureter. In accordance with the above two above, the patients were given the complication of ureteral stone, and the patients were selected to have a case of the urinary calculi.

Methods: using the STROZ company F7.5 ureteral soft mirror, wolff8/9.8 ureteral hard mirror, Cook14F ureter sheath, Olympus imaging system, medical man 100W holmium laser. Under general anaesthesia, the patient is in the transversal position. Hard ureter mirror by distorted or stricture with holmium laser will explore and calculi after partial break into small blocks, after switching to soft lens to move line located within a larger residual kidney calculi holmium laser lithotripsy. After finding the stone, the $200 \mathrm{mu}$ fiber optic is placed in the soft mirror, using the holmium laser $(0.6 \mathrm{~J} / 20 \mathrm{~Hz})$ for the gravel to the diameter $<3 \mathrm{~mm}$. Big block stone, use a stone basket to take out, the stone break with the flush fluid discharge, partial operation can be excreted by oneself. At the end of the operation, the double $\mathrm{J}$ tube was placed in the tube, and the abdominal flat plate KUB was clearly the effect of the gravel and the double $\mathrm{J}$ tube position, and the two J tubes for two to four weeks. After 1 month, the urology department was reviewed and the renal CT examination was reviewed to understand the condition of water and kidney function.

\section{The Results}

In the 47 cases of this group, 35 of the cases were found to be able to reach the stone in the crushing part of 35 cases, and then the soft mirror was used to locate the large residual stones in the kidney, the holmium laser rock. Five cases were placed under the ureter's flexible sheath and the stenosis of the ureter, and the ureteral tube was successfully broken through a narrow, twisted section of the urea stone. Two cases of ureteral tubes were directly placed in the ureteral soft mirror with a narrow diameter of the ureteral tube. In 43 cases, a single stone crushing rate was 91.5\% (43/47), and 2 cases of double $J$ tube were successfully used in the second stage of 2-week. 1 case of hard into the ureter mirror distortion to ureteral segment ureteral perforation, 1 case of ureteral seriously distorted ultra smooth godet and soft ureter mirror failure, are changed to open surgery to take out the parallel ureteral end to end anastomosis. The average surgical time was $65 \mathrm{~min}(47-80 \mathrm{~min})$, and the average length of hospital stay was $4.5 \mathrm{~d}$ (2-9d) after surgery, and 14d-60d after operation. In 21 cases, the appearance of macroscopic hematuria and the disappearance of $1-4 \mathrm{~d}$ were followed by severe complications such as high fever and urinary bladder. Postoperative abdominal plain film review of the 3 cases of residual fragments after ESWL treatment, the big calculus 1 to 3 times, postoperative double $\mathrm{J}$ tube pulling KUB, check after all cases with no more than $4 \mathrm{~mm}$ diameter stone residues have clinical symptoms. In 17 cases before the operation, the water disappeared completely. The remaining 30 cases of renal pelvis were significantly improved.

\section{Discussion}

In recent years, both ureteral and ureteral microscopy have been widely used in the treatment of ureteral stones [2]. Hard ureter mirror is easy to look down her, the advantages of broad vision, especially in large diameter orifices is easy to work in high power holmium laser optical fiber improve the efficiency of gravel, is also conducive to 
put biopsy forceps or foreign body forceps for corresponding operation [3]. Soft ureter mirror more suitable for ureteral section, renal pelvis and calyces on observation and operation, the renal pelvis and calyces on ureteral period of diagnosis and treatment of the disease has an irreplaceable advantages, a new soft ureter mirror is more and more thin, soft, clear, especially combined holmium laser for the treatment of urinary calculi under $2 \mathrm{~cm}$, minimally invasive and efficient [4]. Complexity of ureteral polyps Duan Jieshi often exist on the parcel and calculus incarcerated for a long time, many stone with distal ureteral distorted or narrow, and in the process of ureteroscopy lithotripsy treatment stone easy to shift in the kidney. So simple application soft hard mirror ureter or ureter mirror gravel is insufficient, and the combination of both using the holmium laser lithotripsy is applied to the complexity of ureteral Duan Jieshi treatment effect is better. The holmium laser can effectively crush the urinary stones of various ingredients. Soft ureter mirror with rigid ureteroscopy holmium laser therapy on the complexity of ureteral Duan Jieshi, our experience includes: for the complexity of ureteral Duan Jieshi, ureteral distortion found in distal calculi obstruction position, when the microscopic invisible ureteral lumen is close to the heart part of the distortion, the lens body once by distorting often can explore and stone. In order to minimize the distortion of ureteral tubes, the surgical bed can be changed to a low or high position. The silk is softer in the front of the thread, causing the silk to return in the twisted segment of the ureter. In the case of super slide, we have a high degree of interpolation in the application of ureteral distortion or narrow case. If the ureters are difficult to pass through the diuretic or narrow passage in the hard mirror, they should not be forced into the mirror and use the ureteroscope. Under the guidance of thread, the use of soft lens can bend the head turned to the distortion of the proximal, discern near side ureteral lumen after insert the thread, as far as possible the ureteral twisted section of straight, and then continue placing soft lens. The ideal location of the ureter's soft mirror sheath is in the renal pelvis, and the greatest benefit is the ability to reduce the pressure in the renal pelvis and reduce the risk of infection by flushing. After the detritus of the gravel, the soft mirror sheath was removed. To avoid in the process of soft lens sheath placement causes distorted or narrow ureter perforation, soft lens sheath can be placed in ureteral middle first, and then puts soft mirror lens body within the ureter sheath, lead to distortion under the period of the ureter sheath. In the case of the ureteral cavity, the tube is not covered by the soft mirror sheath, and the ureteral tube is placed directly under the superslide guide. If the upper ureter is distorted and the soft mirror is not inserted directly, the F5 or F6 double $\mathrm{J}$ tube is retained, and the second stage of the 1-2 weeks is the secondary ureteral microscopy. Hard ureter mirror with holmium laser will explore and calculi under partial break into small pieces, after switching to soft lens line will move in the residual stones in the kidney holmium laser lithotripsy, can improve the efficiency of gravel, shorten the operation time [5]. Soft ureter mirror operation channel after placing fiber its front end bending Angle decreased, so the calyx calculi in in situ laser lithotripsy is difficult, the available set of rubble stone basket will be moved to the pelvic again, can improve the clearance rate of stone, at the same time also can reduce the damage of soft lens. After the ureter soft mirror rubble, only the larger stones will be taken out of the stone basket, and the crushed stone will not waste time to set up or rush out. For residual stones with a residual diameter of less than $4 \mathrm{~mm}$ without clinical symptoms, we think long-term follow-up is a good choice.

\section{Conclusions}


Of merger ureteral stricture, the formation of polyps or calculus incarcerated packages and ESWL treatment failure of the complexity of the ureteral Duan Jieshi, soft lens combined with ureter mirror ureter holmium laser lithotripsy is safe, effective, curative effect is a kind of exact intracavitary urology macadam method.

\section{References}

[1] Ureteroscopic management of renal calculi in anomalous kidneys. A.Z. Weizer, W.P. Springhart, W.O. Ekeruo. Urology. 2005

[2] Retrograde ureteroscopic intrarenal surgery for large $(1.6-3.5 \mathrm{~cm})$ upper ureteric/renal calculus. Probhakar M. Indian Journal of Urology. 2010

[3] Flexible Ureterorenoscopy With Holmium Laser in Horseshoe Kidneys [J] . Benoit Molimard, Saeed Al-Qahtani, Amine Lakmichi, Majed Sejiny, Sixtina Gil-Diez de Medina, Xavier Carpentier, Olivier Traxer. Urology. 2010 (6)

[4] Flexible ureteroscopy and holmium laser lithotripsy for treatment of upper urinary tract calculi in patients with autosomal dominant polycystic kidney disease [J]. Liu Yili, Li Yongzhi, Li Ning, Xue Dongwei, Liu Chunlai, Liu Suomin, Wang Ping. Urological Research. 2012 (1)

[5] Successful outcome of flexible ureteroscopy with holmium laser lithotripsy for renal stones $2 \mathrm{~cm}$ or greater $[\mathrm{J}]$. Ryoji Takazawa, Sachi Kitayama, Toshihiko Tsujii. International Journal of Urology. 2012 (3) 